\title{
A Note on T-Duality, Open Strings in B-field Background and Canonical Transformations
}

\author{
M.M. Sheikh-Jabbari 周 \\ The Abdus Salam International Centre for Theoretical Physics \\ Strada Costiera, 11 \\ I-34014, Trieste, Italy
}

\begin{abstract}
In this paper we study T-duality for open strings ending on branes with non-zero B-field on them from the point of view of canonical transformations. For the particular case of type II strings on the two torus we show that the $S l(2, Z)_{N}$ transformations can be understood as a sub-class of canonical transformations on the open strings in the B-field background.
\end{abstract}

\footnotetext{
*E-mail:jabbari@ictp.trieste.it
} 


\section{Introduction}

Recently noncommutative geometry has been shown to be relevant to D-branes in the constant B-field background [1, 2, 3, 4, 5]. In [1], it was conjectured that a light cone formulation of M-theory with the non-zero constant 3-from background on a three torus is given by the SYM on noncommutative two torus, $T_{\theta}^{2}$, and, considering this background is crucial to recover the U-duality group of M-theory on $T^{3}, S L(3, Z) \times S l(2, Z)_{N}$, where the deformation parameter of the noncommutative torus , $\theta$, plays the role of $S l(2, Z)_{N}$ metric in the zero volume limit.

In a string theoretic discussion it was shown that quantizing the open strings with the mixed boundary conditions, the coordinates of end points of such open strings, $x_{i}$, which live on a D-brane with a non-zero B-field background are really noncommuting [3, 6, 7] and:

$$
\left[x_{i}, x_{j}\right]=2 i \alpha^{\prime}\left(B\left(1-B^{2}\right)^{-1}\right)_{i j} .
$$

Besides the noncommutative structure, it was shown that the T-duality group of type II theories on $T^{2}, S O(2,2 ; Z)$ can be written as $S l(2, Z) \times S l(2, Z)_{N}$, where the first is the mapping class group of the torus and the second acts on the Kahler moduli of the torus [3, 田.

On the other hand there have been many suggestions in literature trying to explain Tduality as particular canonical transformations [8, 9, 10]. This idea was initially studied for the closed strings in general backgrounds and was extended to the open strings with Dirichlet boundaries, D-branes. However the case of D-brane bound states, i.e. , open strings ending on the branes with non-zero B-field [11] has not been elaborated on well.

In this note we will study the behaviour of open strings with different boundary conditions under the proper canonical transformations and show that the $S l(2, Z)_{N}$ part of T-duality is really a sub-class of these canonical transformations.

Open string in B-field backgrounds

We start with the usual $\sigma$-model action describing the open strings in B-field backgrounds:

$$
S=\frac{1}{4 \pi \alpha^{\prime}} \int_{\Sigma} d^{2} \sigma\left[\eta_{\mu \nu} \partial_{a} X^{\mu} \partial_{b} X^{\nu} g^{a b}+\epsilon^{a b} B_{\mu \nu} \partial_{a} X^{\mu} \partial_{b} X^{\nu}+\frac{1}{2 \pi \alpha^{\prime}} \oint_{\partial \Sigma} d \tau A_{i} \partial_{\tau} \zeta^{i}\right.
$$

where $A_{\mu}$, is the $U(1)$ gauge field living on the D-brane and $\zeta^{i}$ its internal coordinates. The action is invariant under the combined gauge transformation [2]

$$
\begin{gathered}
B_{\mu \nu} \rightarrow B_{\mu \nu}+\partial_{\mu} \Lambda_{\nu}-\partial_{\nu} \Lambda_{\mu} \\
A_{\mu} \rightarrow A_{\mu}-\Lambda_{\mu} .
\end{gathered}
$$


The gauge invariant field strength is then

$$
\mathcal{F}_{\mu \nu}=B_{\mu \nu}-F_{\mu \nu} \quad, \quad F_{\mu \nu}=\partial_{[\mu} A_{\nu]}
$$

So we can always choose $\Lambda_{\mu}$ so that $\mathcal{F}_{\mu \nu}=B_{\mu \nu}$, and hereafter we will work in this gauge.

Variation of the action $S$, leads to the following mixed boundary conditions

$$
\partial_{\sigma} X^{\mu}+\mathcal{F}_{\nu}^{\mu} \partial_{\tau} X^{\nu}=0
$$

or in terms of the conjugate canonical momenta, $P_{\mu}$ :

$$
\partial_{\sigma} X^{\mu}+\alpha^{\prime} f^{\mu \nu} P_{\nu}=0
$$

with

$$
f_{\mu \nu}=\left(\mathcal{F}\left(1-\mathcal{F}^{2}\right)^{-1}\right)_{\mu \nu}
$$

Since we will mostly consider the compactifications of $X^{1}$ and $X^{2}$ directions on two torus, for simplicity we assume that all the $\mathcal{F}$ components except $\mathcal{F}_{12}=F$ to be zero and in this case I

$$
f_{\mu \nu}=\epsilon_{\mu \nu} f, \quad f=\frac{F}{1+F^{2}} .
$$

$f$ is the deformation parameter of the noncommutative tours.

Let us return to the question of canonical transformations . It is well known that, although the canonical transformations do not change the equations of motion, they alter the boundary conditions and hence in our case we expect the boundary conditions, or equivalently the parameter $f$, to be changed with canonical transformations. But, we will momentarily show that for any linear canonical transformation one can find a transformed $f$, so that the form of the boundary conditions remain invariant. This argument can be justified as follows: Boundary conditions can be treated as the constraints which are of second class [12] and hence the canonical transformations are those under which the Dirac brackets are invariant. The above requirement is satisfied if we also transform $f$ in a proper way.

Since we are interested in the two torus case, we consider the canonical transformations which reproduce the $S l(2, Z)_{N}$

a)

\footnotetext{
${ }^{\dagger}$ If we consider the torus radii, $R_{1}$ and $R_{2}$, we find $f=\frac{F}{V^{2}+F^{2}}$, where $V=R_{1} R_{2}$.
} 


$$
\left\{\begin{array}{c}
X_{i} \rightarrow \tilde{X}_{i}=\alpha^{\prime} \int^{\sigma} P_{i} d \sigma \\
P_{i} \rightarrow \tilde{P}_{i}=\partial_{\sigma} X_{i}, \quad i=1,2
\end{array}\right.
$$

together with $\mathrm{f}$

$$
f \rightarrow \tilde{f}=\frac{-1}{f} .
$$

One can easily show that the above transformations do not change the form of equations of motion and the boundary conditions,

$$
\partial_{\sigma} X^{\mu}+\alpha^{\prime} f^{\mu \nu} P_{\nu}=0 \rightarrow \partial_{\sigma} \tilde{X}^{\mu}+\alpha^{\prime} \tilde{f}^{\mu \nu} \tilde{P}_{\nu}=0
$$

In other words under the above transformations the Dirac bracket introduced in [7, 12] are invariant. It is worth noting that the above transformations are exactly the same as the transformations introduced in [8] for the closed strings.

b)

$$
\left\{\begin{array}{c}
X_{i} \rightarrow \tilde{X}_{i}=X_{i}+\epsilon_{i j} \alpha^{\prime} \int^{\sigma} P_{j} d \sigma \\
P_{i} \rightarrow \tilde{P}_{i}=P_{i}, \quad i=1,2,
\end{array}\right.
$$

together with the proper metric transformations (the usual T-duality transformations), and the $f \rightarrow \tilde{f}=f+1$, are also the symmetry of the open string action in the same sense as the case a).

As we see the $S l(2, Z)_{N}$ transformations which act on the Kahler structure of the torus, $\rho=i V+f$, can be generated by the transformations on canonical variables and also the $f$, background field. All the above arguments can easily be generalized to the case of $T^{n}$.

\section{Concluding Remarks}

In this note we showed that the idea of treating T-duality group of the perturbative closed string theories (type II strings) as canonical transformations of the $\sigma$-model can be extended to the strong couplings, using the fact that at strong coupling type II theories are perturbatively governed by the open strings ending on the D-branes and their bound states. In this case although the open string boundary conditions (or equivalently the brane structure

\footnotetext{
$\ddagger$ For the case of the torus we should take $\alpha^{\prime}$ to $\alpha^{\prime} R_{i}^{2}$ and the metric is changed as the usual T-dualities.
} 
we have) change under canonical transformations, we argued that by a proper change of parameters we can make our theory to be invariant under such transformations, which exactly lead to the $S l(2, Z)_{N}$ part of the expected T-duality group. On the other hand it has been argued that this duality can be realized as the Morita equivalence of the noncommutative gauge bundles governing the low energy effective theory of the open strings we considered here. So it is an interesting question to study Morita equivalence in the light of canonical transformations.

One can address the same procedure in the path integral formulation too. In the path integral, canonical transformations are translated to changing the operators by similarity transformations. This point of view has recently been used for quantizing the discretized M2-branes in a three from field background [13]. In this picture $\mathrm{T}$ (or $\mathrm{U}$ )-duality is given by a special class of the unitary transformations which are characterized by C-field. This question from the string theory point of view can be understood through the DVV [14] string matrix theory.

\section{Acknowledgements}

I would like to thank H. Arfaei and F. Ardalan for many fruitful discussions.

\section{References}

[1] A. Connes, M.R. Douglas, A. Schwarz, "Noncommutative Geometry and Matrix Theory: Compactification on Tori", JHEP 9802 (1998) 003, hep-th/9711162.

[2] M. R. Douglas, C. Hull, "D-branes and Noncommutative Torus", JHEP 9802 (1998) 008, hep-th/9711165.

Y.-K. E. Cheung, M. Krogh, "Noncommutative Geometry From 0-Branes in a Background $B$ Field", hep-th/9803031.

F. Ardalan, H. Arfaei, M. M. Sheikh-Jabbari, "Mixed Branes and M(atrix) Theory on Noncommutative Torus", hep-th/9803067.

[3] F. Ardalan, H. Arfaei, M. M. Sheikh-Jabbari, "Noncommutative Geometry form Strings and Branes", JHEP 02 (1999) 016, hep-th/9810072.

[4] C. Hofman and E. Verlinde, "U-duality of Born-Infeld on the Noncommutative Two Torus", JHEP 9812 (1998) 010, hep-th/9810116. 
[5] N. Seiberg, E. Witten, "String Theory and Noncommutative Geometry", JHEP 09 (1999) 032, hep-th/9908142.

[6] C-S. Chu and P-M. Ho, "Noncommutative Open Strings and D-branes", Nucl. Phys. B550 (1999) 151, hep-th/9812219.

[7] F. Ardalan, H. Arfaei, M. M. Sheikh-Jabbari, "Dirac Quantization of Open Strings and and Noncommutativity in Branes", hep-th/9906161.

C-S. Chu, P.M. Ho, "Constrained Quantization of Open Strings in Background B-Field and Noncommutative D-Branes", hep-th/9906192.

[8] A. Giveon, E. Rabinovici and G. Veneziano, Nucl. Phys. B322 (1989) 167.

E. Alvarez, L. Alvarez-Gaume' and Y. Lozano, Phys. Lett. B336 (1994) 183.

A. Giveon, M. Poratti and E. Rabinovici, Phys. Rep. 244 (1994) 77.

[9] H. Dorn, H.-J. Otto, Phys. Lett. B381 (1996) 81.

[10] Y. Lozano, Mod. Phys. Lett. A11 (1996) 2893, hep-th/9610024 and references therein.

[11] E. Gava, K.S. Narain, M.H. Sarmadi, "On the Bound State of p- and (p+2)-Branes", Nucl. Phys. B504 (1997) 214, hep-th/9704006.

H. Arfaei, M.M. Sheikh-Jabbari, "Mixed Boundary Conditions and Brane-String Bound States", Nucl. Phys. B526 (1998) 278, hep-th/9709054.

M.M. Sheikh-Jabbari, "More on Mixed Boundary Conditions and D-brane Bound States", Phys. Lett. B425 (1998) 48.

[12] M.M. Sheikh-Jabbari and A. Shirzad, "Boundary Conditions as Dirac Constraints ", hep-th/9907055.

[13] C-S. Chu, P-M. Ho, M. Li, "Matrix Theory in a Constant C Field Background", hepth/9911153.

[14] R. Dijgraaf, E. Verlinde, H. Verlinde, "Matrix String Theory", Nucl. Phys. B500 (1997) 43, hep-th/9703030. 